\title{
NUMERICAL SOLUTION OF A NONLOCAL FRACTIONAL BOUNDARY VALUE PROBLEM BY HDG METHOD
}

\author{
Mehmet Fatih Karaaslan \\ Department of Statistics, Faculty of Arts and Science, Yildiz Technical University, Istanbul, Turkey \\ mfatih@yildiz.edu.tr
}

\begin{abstract}
This paper is concerned with numerically solving of a nonlocal fractional boundary value problem (NFBVP) by hybridizable discontinuous Galerkin method (HDG). The HDG methods have been successfully applied to ordinary or partial differential equations in an efficient way through a hybridization procedure. These methods reduce the globally coupled unknowns to approximations at the element boundaries. The stability parameter has to be suitably defined to guarantee the existence and uniqueness of the approximate solution. Some numerical examples are given to show the performance of the HDG method for NFBVP.
\end{abstract}

Keywords: Hybridizable discontinuous Galerkin methods, nonlocal fractional boundary value problem, stability parameter, hybridization.

\section{Introduction}

Consider a fractional differential equation with nonlocal boundary conditions as follows [1]

$$
\begin{gathered}
{ }^{C} D^{\alpha} u(x)+a_{0}(x) u^{\prime}(x)+a_{1}(x) u(x)=f(x), \text { in } \Omega=(0,1) \\
u=g, \text { on } \partial \Omega=\{0,1\}
\end{gathered}
$$

Where $1<\alpha<2, g$ is a given function such that $g(0)=0, g(1)=\sum_{i=1}^{m} \beta_{i} u\left(\mu_{i}\right), 0<\mu_{i}<1$, $a_{j}(j=0,1)$, and $f$ are given functions such that satisfy the existence and uniqueness of the solutions of the problem. Fractional differential equations arise in modeling many natural phenomena of physics, engineering, electrodynamics of complex medium, aerodynamics, porous media, continuum and statistical mechanics [2-7]. The multipoint boundary conditions arise from many phenomena such as thermodynamics, elasticity, and wave propagation of applied sciences [8] and the references therein. These boundary conditions can be interpreted 
as the controllers at the end of the points distribute or add energy with respect to censors settled at intermediate points. Some basic studies and recent researches on the NFBVP s can be found in [9-15].

Benchohra et al. [16] established sufficient conditions for the existence results of NFBVP with Caputo derivative

$$
\begin{gathered}
{ }^{C} D^{\alpha} u(x)=f(x, u(x)), 0 \leq x \leq T, 1<\alpha \leq 2, \\
u(0)=g(u), u(T)=u_{T}
\end{gathered}
$$

where $f:[0, T] \mathrm{x} R \rightarrow R$ and $g: C(J, R) \rightarrow R$ are continuous functions. Bai [17] studied the existence and uniqueness solution of the following nonlocal problem

$$
\begin{gathered}
D^{\alpha} u(x)+f(x, u(x))=0,0<x<1, \\
u(0)=0, \beta u(\eta)=u(1),
\end{gathered}
$$

where $1<\alpha \leq 2,0<\beta \eta^{\alpha-1}<1,0<\eta<1$ and $f$ is a continuous function on $[0,1] \times[0, \infty]$.

El-Sayed and Bin-Thaer [18] obtained the existence of at least one solution for nonlocal multipoint boundary value problem as follows

$$
\begin{array}{cc}
u^{\prime \prime}+f\left(x,{ }^{C} D^{\alpha} u(x)\right)=0, & 0<x<1,0<\alpha<1, \\
u(0)=0, u(1)=\sum_{j=1}^{k} a_{j} u\left(\tau_{j}\right), \tau_{j} \in(a, b) \subset(0,1) .
\end{array}
$$

$\mathrm{Li}$ and $\mathrm{Wu}$ [19] proposed a new numerical method based on the reproducing kernel method for the following NFBVP

$$
\begin{gathered}
{ }^{C} D^{\alpha} u(x)+a(x) u^{\prime}(x)+b(x) u(x)=f(x), x \in[0,1], 1<\alpha \leq 2, \\
\beta_{1} u=\mu_{1}, \beta_{2} u=\mu_{2}
\end{gathered}
$$

where $\beta_{1} u=\mu_{1}$ and $\beta_{2} u=\mu_{2}$ are linear nonlocal boundary conditions, $a(x), b(x) \in W^{1}[0,1]$, $u(x) \in W^{4}[0,1]$, the existence and uniqueness of the solution are provided for a suitable $f(x)$.

Cockburn et al. [16] firstly presented the HDG methods in the framework of second order elliptic problems. These methods involve hybridized mixed, continuous Galerkin, nonconforming, and HDG methods. The HDG methods have an efficient convergence results for the ordinary or partial differential equations and a built-in stabilization mechanism which do not degrade their accuracy [20-23].

The application of the HDG methods for fractional differential equations is limited in the literature. Cockburn and Mustapha [24] used the HDG method for numerically solving subdiffusion fractional model problem for $-1<\alpha<0$. Then, Mustapha et al. [25] studied the method for the spatial discretization of time fractional diffusion equation with Caputo derivative of order $0<\alpha<1$. Karaaslan et al. [26, 27] obtained the numerical solution of the Bagley-Torvik equation and a class of fractional boundary value problem (FBVP) with $1<\alpha<2$ and $0<\beta<1$ by HDG method. 
The aim of this paper is to derive an efficient numerical solution of (1)-(2) problem by HDG method. To the best of my knowledge, this is the first time an HDG method is applied to the NFBVPs in a systematic way.

The organization of this paper is as follows. In Section 2, the HDG method is presented with its basis components for the NFBVP. The characterization of the HDG approximation is given in Section 3. The convergence results of the method are given on numerical examples in Section 4.

\section{The HDG methods}

The HDG method is applied to the (1)-(2) problem by defining Caputo fractional derivate with an operator $T$ as follows

$$
{ }_{0}^{C} D_{x}^{\alpha} u=\frac{1}{\Gamma(2-\alpha)} \int_{0}^{x} \frac{u^{\prime \prime}(\xi)}{(x-\xi)^{\alpha-1}} d \xi:=T\left[u^{\prime \prime}(x)\right] .
$$

Using two unknown variables $p$ and $q$ in (1), the HDG formulation is obtained as follows

$$
\begin{array}{lll}
q(x)+u^{\prime}(x) & =0, & \text { in } \Omega, \\
p(x)+q^{\prime}(x) & =0, & \text { in } \Omega, \\
a_{0} T[p(x)]-a_{1} q(x)+a_{2} u(x) & =f(x), & \text { in } \Omega .
\end{array}
$$

Given a positive integer $N, \Omega$ is divided into $N$ elements by setting

$$
\Omega_{h}:=\left\{I_{k}=\left(x_{k-1}, x_{k}\right) \mid 0=x_{0}<x_{1}<\cdots<x_{N-1}<x_{N}=1\right\} .
$$

The set of nodes and interior nodes are defined as $\varepsilon_{h}:=\left\{x_{0}, x_{1}, \ldots, x_{N}\right\}, \varepsilon_{h}^{0}:=\varepsilon_{h} \backslash \partial \Omega$; and $\partial \Omega_{h}:=\left\{\partial K: K \in \Omega_{h}\right\}$, respectively. For $K \in \Omega_{h}, h_{K}$ is the length of $K, h:=\max _{K \in \Omega_{h}}\left\{h_{K}\right\}$. Let $P^{k}(K)$ denote the set of polynomials of degree less than or equal to $k \geq 0$ that is a given polynomial degree on $K$. The space of piecewise polynomials of degree $k$ on $\Omega$ is defined as follows

$$
V_{h}^{k}:=\left\{v: \Omega_{h} \rightarrow \mathbb{R}:\left.v\right|_{K} \in P^{k}(K) \text { for all } K \in \Omega_{h}\right\}
$$

and

$$
L_{0}^{2}\left(\varepsilon_{h}\right):=\left\{m \in L^{2}\left(\varepsilon_{h}\right): m=0 \text { on } \partial \Omega\right\}
$$

where $L^{2}\left(\varepsilon_{h}\right)$ is a copy of the $\mathbb{R}^{N+1}$. 
The HDG method find a numerical approximation given as $\left(q_{h}, p_{h}, u_{h}, \hat{u}_{h}\right)$ to the $\left(q, p, u, u_{\varepsilon_{h}}\right)$ that is the exact solution of (12) where the space is defined as $V_{h}^{k} \times V_{h}^{k} \times V_{h}^{k} \times L^{2}\left(\varepsilon_{h}\right)$. This approximate solution is defined for $(v, w, z, \mu) \in V_{h}^{k} \times V_{h}^{k} \times V_{h}^{k} \times L_{0}^{2}\left(\varepsilon_{h}\right)$ as follows

$$
\begin{aligned}
&\left(q_{h}, v\right) \quad-\left(u_{h}, v^{\prime}\right)+<\hat{q}_{h}, v \cdot n>=0, \\
&\left(p_{h}, w\right)-\left(q_{h}, w^{\prime}\right)+<\hat{q}_{h}, w \cdot n>=0, \\
& a_{0}\left(T\left[p_{h}\right], z\right)-a_{1}\left(q_{h}, z\right)+ a_{2}\left(u_{h}, z\right)=(f, z), \\
&<\hat{q}_{h} \cdot n, \mu>=0,
\end{aligned}
$$

The boundary condition (2) is imposed by requiring that

$$
\hat{u}_{h}=u_{D} \quad \text { on } \partial \Omega
$$

and the numerical trace $\hat{q}_{h}$ is given as follows

$$
\hat{q}_{h}=q_{h}+\tau\left(u_{h}-\hat{u}_{h}\right) n
$$

where $\tau$ is a stability parameter of the linear system that is a nonnegative function on $\partial \Omega_{h}$. (13), (14) and (15) construct the framework of the HDG method.

\section{Characterization of the HDG approximation}

This section are first give the local solvers that form the basis of the HDG method. The first local solver is defined on $K \in \Omega_{h}$ as the mapping $\mu \in L^{2}(\partial K) \mapsto(Q \mu, P \mu, U \mu) \in\left[P^{k}(K)\right]^{3}$ where

$$
\begin{aligned}
& (Q \mu, v)_{K} \quad-\left(U \mu, v^{\prime}\right)_{K} \quad=-\langle\mu, v n\rangle_{\partial K}, \\
& \left(-w^{\prime}, Q \mu\right)_{K}+(P \mu, w)_{K}+\langle w, \hat{Q} \mu . n\rangle_{\partial K}=0, \\
& a_{0}(T[P \mu], z)_{K}-a_{1}(Q \mu, z)_{K}+a_{2}(U \mu, z)_{K}=0,
\end{aligned}
$$

for all $v, w, z \in P^{k}(K)$ where $\hat{Q} \mu=Q \mu+\tau(U \mu-\mu) n$, on $\partial K$.

The second local solver is defined on the element $K \in \Omega_{h}$ as the mapping $f \in L^{2}(K) \mapsto(Q f, P f, U f) \in\left[P^{k}(K)\right]^{3}$ where

$$
\begin{array}{lll}
(Q f, v)_{K} & -\left(U f, v^{\prime}\right)_{K} & =0, \\
\left(-w^{\prime}, Q f\right)_{K} & +(P f, w)_{K}+\langle w, Q f f . n\rangle_{\partial K} & =0, \\
a_{0}(T[P f], z)_{K}-a_{1}(Q f, z)_{K}+a_{2}(U f, z)_{K} & =(f, z)_{K}
\end{array}
$$

for all $v, w, z \in P^{k}(K)$ where $\hat{Q} f=Q f+\tau(U f-0) n, \quad$ on $\partial K$. 
The function $u_{D}$, as well as any other function defined only on $\partial \Omega$ is extended to $\varepsilon_{h}$ by zero.

Also,

$$
\lambda_{h}:= \begin{cases}\hat{u}_{h} & \text { on } \partial \Omega_{h} \backslash \partial \Omega, \\ 0 & \text { on } \partial \Omega,\end{cases}
$$

and $\hat{u}_{h}=\lambda_{h}+u_{D}$ where $\lambda_{h} \in L_{0}^{2}\left(\varepsilon_{h}\right)$.

Theorem 3.1 The approximate solution $\left(q_{h}, p_{h}, u_{h}, \lambda_{h}\right) \in\left[V_{h}^{k}\right]^{3} \times L_{0}^{2}\left(\varepsilon_{h}\right)$ obtained by the HDG method (13) can be written with respect to the local solvers as

$$
\begin{aligned}
& q_{h}=Q \lambda_{h}+Q u_{D}+Q f, \\
& p_{h}=P \lambda_{h}+P u_{D}+P f, \\
& u_{h}=U \lambda_{h}+U u_{D}+U f,
\end{aligned}
$$

where $\lambda_{h}$ satisfies

$$
a_{h}\left(\lambda_{h}, m\right)=b_{h}(m), \quad \text { for all } m \in L_{0}^{2}\left(\varepsilon_{h}\right),
$$

where

$$
\begin{aligned}
& a_{h}\left(\lambda_{h}, m\right)=-<\hat{Q} \lambda_{h} m n>_{\partial \Omega_{h}}, \\
& b_{h}(m)=<\hat{Q} u_{D}+\hat{Q} f m n>_{\partial \Omega_{h}} .
\end{aligned}
$$

Proof. It is clearly seen as a reference to [20] and [28] rewriting (19) with respect to the last equation called as conservativity condition in (13).

Remark 2.2 The logarithm of the ratio of the two consecutive errors for the two HDG approximations with consecutive meshes are given as convergence order of the approximate solution and

$$
\left\|\varphi-\varphi_{h 0} \leq C h^{\eta}\right\|
$$

where $\eta$ is convergence order, $C$ is a constant number independent of $h$. Also,

$$
\left\|u-\hat{u}_{h}\right\|_{\infty}=\max _{e \in \varepsilon_{h}}\left|\left(u-\hat{u}_{h}\right)(e)\right|
$$

and the stability parameter $\tau=1$ is taken on $\partial \Omega_{h}$ in each examples.

\section{Numerical examples}

In this section, two numerical examples are given to test the convergence order and accuracy of the HDG approximation for NFBVPs using MATLAB 2014a.

Example 4.1 Consider the following nonlocal fractional boundary value problem [19, 29] 


$$
\begin{aligned}
& { }^{C} D^{\alpha} u(x)+\delta u(x)=f(x), \quad \Omega=(0,1), 1 \leq \alpha<2 \\
& u(0)=0, \quad u(1)=\xi u(\eta), \partial \Omega=\{0,1\}
\end{aligned}
$$

where $\alpha=\frac{3}{2}, \delta=\frac{e^{-3 \pi}}{\sqrt{\pi}}, \xi=-\frac{125}{196}, \eta=\frac{2}{5}, u(1)=-\frac{1}{40}$,

$f(x)=\frac{e^{-3 \pi}}{40 \sqrt{\pi}}\left(x^{2}\left(40 x^{2}-74 x+33\right)+4 e^{3 \pi} \sqrt{x}\left(128 x^{2}-148 x+33\right)\right)$ and the exact solution is given as $u(x)=x^{2}\left(x^{2}-\frac{37}{20} x+\frac{33}{40}\right)$. The error value and convergence order for $L^{2}$-norm are presented in Table 1. For $k=2$ and $k=3, u_{h}$ and $q_{h}$ converge with order $k+1$. For $k=2$, $p_{h}$ and $\hat{u}_{h}$ converge with order $k-1$ whereas the HDG method captures the exact solution (up to the machine accuracy) for $p_{h}$ and $\hat{u}_{h}$ in $k=3$. However, the exact solution is captured for $u_{h}$ and $q_{h}$ in $k=4$.

Table 1. Convergence order and error result for Example 1.

\begin{tabular}{|c|c|c|c|c|c|c|c|c|c|}
\hline \multicolumn{2}{|c|}{} & \multicolumn{2}{c|}{$\left\|u-u_{h}\right\|_{0, \Omega}$} & \multicolumn{2}{|c|}{$\left\|q-q_{h}\right\|_{0, \Omega}$} & \multicolumn{2}{c|}{$\left\|p-p_{h}\right\|_{0, \Omega}$} & \multicolumn{2}{c|}{$\left\|u-\hat{u}_{h}\right\|_{L^{2}\left(\varepsilon_{h}\right)}$} \\
\hline $\mathbf{k}$ & $\mathbf{N}$ & Error & Order & Error & Order & Error & Order & Error & Order \\
\hline \multirow{4}{*}{2} & 8 & $1.78 \mathrm{e}-04$ & 2.99 & $1.56 \mathrm{e}-04$ & 2.99 & $3.87 \mathrm{e}-07$ & 1.15 & $3.67 \mathrm{e}-08$ & 0.87 \\
\cline { 2 - 12 } & 16 & $2.24 \mathrm{e}-05$ & 2.99 & $1.95 \mathrm{e}-05$ & 3.00 & $1.90 \mathrm{e}-07$ & 1.03 & $1.86 \mathrm{e}-08$ & 0.99 \\
\cline { 2 - 11 } & 32 & $2.80 \mathrm{e}-06$ & 3.00 & $2.44 \mathrm{e}-06$ & 3.00 & $9.45 \mathrm{e}-08$ & 1.01 & $9.32 \mathrm{e}-09$ & 0.99 \\
\cline { 2 - 11 } & 64 & $3.51 \mathrm{e}-07$ & 3.00 & $3.05 \mathrm{e}-07$ & 3.00 & $4.73 \mathrm{e}-08$ & 1.00 & $4.67 \mathrm{e}-09$ & 1.00 \\
\cline { 2 - 11 } & 128 & $4.39 \mathrm{e}-08$ & 3.00 & $3.85 \mathrm{e}-08$ & 2.99 & $2.36 \mathrm{e}-08$ & 1.00 & $2.34 \mathrm{e}-09$ & 1.00 \\
\hline \multirow{4}{*}{3} & 8 & $1.16 \mathrm{e}-06$ & 4.00 & $1.31 \mathrm{e}-06$ & 3.99 & $4.08 \mathrm{e}-11$ & & $3.86 \mathrm{e}-12$ & \\
\cline { 2 - 11 } & 16 & $7.27 \mathrm{e}-08$ & 4.00 & $8.20 \mathrm{e}-08$ & 3.99 & $2.92 \mathrm{e}-11$ & & $9.33 \mathrm{e}-13$ & \\
\cline { 2 - 11 } & 32 & $4.54 \mathrm{e}-09$ & 4.00 & $5.14 \mathrm{e}-09$ & 4.00 & $2.69 \mathrm{e}-10$ & & $5.42 \mathrm{e}-12$ & \\
\cline { 2 - 11 } & 64 & $2.86 \mathrm{e}-10$ & 3.99 & $3.45 \mathrm{e}-10$ & 3.90 & $2.15 \mathrm{e}-09$ & & $4.95 \mathrm{e}-11$ & \\
\hline \multirow{4}{*}{4} & 4 & $1.93 \mathrm{e}-13$ & & $6.55 \mathrm{e}-13$ & & $3.61 \mathrm{e}-12$ & & $2.95 \mathrm{e}-13$ & \\
\cline { 2 - 11 } & 8 & $4.89 \mathrm{e}-12$ & & $2.08 \mathrm{e}-11$ & & $1.75 \mathrm{e}-10$ & & $7.61 \mathrm{e}-12$ & \\
\cline { 2 - 10 } & 16 & $7.26 \mathrm{e}-11$ & & $3.30 \mathrm{e}-10$ & & $5.70 \mathrm{e}-09$ & & $1.16 \mathrm{e}-10$ & \\
\hline
\end{tabular}

Example 4.2 Consider a fractional differential equation with three-point boundary conditions

$$
\begin{aligned}
& { }^{C} D^{\alpha} u(x)+u^{\prime}(x)+2 u(x)=f(x), \quad \Omega=(0,1), \\
& u(0)=0, u(1)=\frac{1}{8}+2 u\left(\frac{1}{2}\right)+\frac{31}{49} u\left(\frac{7}{8}\right)
\end{aligned}
$$

where the fractional order $\alpha=1.3, f(x)=2 x^{2}+2 x+\frac{\Gamma(3)}{\Gamma(1.7)} x^{0.7}$, and the exact solution is given as $u(x)=x^{2}$. Table 2 shows that for $k=1$, the convergence order of the $u_{h}, q_{h}$, and the numerical trace $\hat{u}_{h}$ is 1 while $p_{h}$ slowly convergences to 1 unlike the others. For $k=2$, the numerical solution $u_{h}, q_{h}, p_{h}$, and the numerical trace $\hat{u}_{h}$ capture the exact solution (up to the machine accuracy). 
Table 2. Convergence order and error result for Example 2.

\begin{tabular}{|c|c|c|c|c|c|c|c|c|c|}
\hline \multicolumn{2}{|c|}{} & \multicolumn{2}{c|}{$\left\|u-u_{h}\right\|_{0, \Omega}$} & \multicolumn{2}{c|}{$\left\|q-q_{h}\right\|_{0, \Omega}$} & \multicolumn{2}{c|}{$\left\|p-p_{h}\right\|_{0, \Omega}$} & \multicolumn{2}{c|}{$\left\|u-\hat{u}_{h}\right\|_{L^{2}\left(\varepsilon_{h}\right)}$} \\
\hline $\mathbf{k}$ & $\mathbf{N}$ & Error & Order & Error & Order & Error & Order & Error & Order \\
\hline \multirow{4}{*}{1} & 8 & $1.79 \mathrm{e}-03$ & 1.54 & $4.36 \mathrm{e}-03$ & 1.04 & $2.71 \mathrm{e}-02$ & 0.48 & $1.90 \mathrm{e}-03$ & 0.99 \\
\cline { 2 - 11 } & 16 & $7.78 \mathrm{e}-04$ & 1.20 & $2.26 \mathrm{e}-03$ & 0.95 & $1.83 \mathrm{e}-02$ & 0.57 & $9.81 \mathrm{e}-04$ & 0.95 \\
\cline { 2 - 11 } & 32 & $3.76 \mathrm{e}-04$ & 1.05 & $1.17 \mathrm{e}-03$ & 0.95 & $1.16 \mathrm{e}-02$ & 0.65 & $5.02 \mathrm{e}-04$ & 0.97 \\
\cline { 2 - 11 } & 64 & $1.87 \mathrm{e}-04$ & 1.01 & $5.95 \mathrm{e}-04$ & 0.97 & $7.15 \mathrm{e}-03$ & 0.70 & $2.54 \mathrm{e}-04$ & 0.98 \\
\cline { 2 - 10 } & 128 & $9.36 \mathrm{e}-05$ & 1.00 & $3.00 \mathrm{e}-04$ & 0.99 & $4.31 \mathrm{e}-03$ & 0.73 & $1.27 \mathrm{e}-04$ & 0.99 \\
\hline \multirow{3}{*}{2} & 4 & $4.50 \mathrm{e}-15$ & & $1.21 \mathrm{e}-14$ & & $1.38 \mathrm{e}-13$ & & $4.41 \mathrm{e}-15$ & \\
\cline { 2 - 10 } & 8 & $7.56 \mathrm{e}-15$ & & $2.59 \mathrm{e}-14$ & & $3.30 \mathrm{e}-13$ & & $9.21 \mathrm{e}-15$ & \\
\cline { 2 - 10 } & 16 & $8.70 \mathrm{e}-15$ & & $4.22 \mathrm{e}-14$ & & $5.07 \mathrm{e}-13$ & & $1.53 \mathrm{e}-14$ & \\
\hline
\end{tabular}

\section{Conclusion}

In this paper, the numerical solution of a NFBVP is investigated using HDG method. The HDG method captures the exact solution (up to the machine accuracy) of these problems by increasing the degree of the polynomial basis functions. The convergence order and error show the effective performance of the HDG method on the problem under consideration.

\section{References}

[1] Geng, Fazhan, and Minggen Cui. "A reproducing kernel method for solving nonlocal fractional boundary value problems." Applied Mathematics Letters 25, no.2 (2012): 818-823.

[2] Hilfer, Rudolf. Applications of fractional calculus in physics. ed. Singapore: World Scientific, 2000.

[3] Kilbas, Anatoly A., Hari M. Srivastava, and Juan J. Trujillo. Theory and Applications of Fractional Differential Equations. Amsterdam: Elsevier, 2006.

[4] Podlubny, Igor. Fractional differential equations. San Diego: Academic Press, 1999.

[5] Samko, Stefan G., Anatoly A. Kilbas, and Oleg I. Marichev. Fractional integrals and derivatives-Theory and Applications. Amsterdam: Gordon and Breach Science Publishers, 1993.

[6] Tenreiro Machado, J. A., Manuel F. Silva, Ramiro S. Barbosa, Isabel S. Jesus, Cecilia M. Reis, Maria G. Marcos, and Alexandra F. Galhano. "Some applications of fractional calculus in engineering." Mathematical Problems in Engineering 2010 (2010): 34.

[7] Zhou, Yong, Jinrong Wang, and Lu Zhang. Basic theory of fractional differential equations. Singapore: World Scientific, 2016.

[8] Ma, Ruyun. "Multiple positive solutions for nonlinear m-point boundary value problems.” Applied Mathematics and Computation 148, no.1 (2004): 249-262.

[9] Ahmad, Bashir, and Juan J. Nieto. "Existence of solutions for nonlocal boundary value problems of higher-order nonlinear fractional differential equations." Abstract and Applied Analysis 2009 (2009): 9. 
[10] Khalil, Hammad, Rahmat Ali Khan, Dumitru Baleanu, and Samir H. Saker. "Approximate solution of linear and nonlinear fractional differential equations under m-point local and nonlocal boundary conditions." Advances in Difference Equations 2016, no.1 (2016): 1-28.

[11] Moshinsky, M. "Sobre los problemas de condiciones a la frontiera en una dimension de caracteristicas discontinuas.” Bol Soc Mat Mexicana 7 (1950): 1-25.

[12] Nyamoradi, Nemat. "Existence of solutions for multi-point boundary value problems for fractional differential equations." Arab Journal of Mathematical Sciences 18, no.2 (2012): 165-175.

[13] Shu, Xiao-Bao, and Qianqian Wang. "The existence and uniqueness of mild solutions for fractional differential equations with nonlocal conditions of order $1<\alpha<2$." Computers \& Mathematics with Applications 64, no.6 (2012): 2100-2110.

[14] ur Rehman, Mujeeb, and Rahmat Ali Khan. "Existence and uniqueness of solutions for multi-point boundary value problems for fractional differential equations." Applied Mathematics Letters 23, no.9 (2010): 1038-1044.

[15] Zhong, Wenyong, and Wei Lin. "Nonlocal and multiple-point boundary value problem for fractional differential equations." Computers \& Mathematics with Applications 59, no.3 (2010): 1345-1351.

[16] Benchohra, M., S. Hamani, and S. K. Ntouyas. "Boundary value problems for differential equations with fractional order and nonlocal conditions." Nonlinear Analysis: Theory, Methods \& Applications 71, no.7 (2009): 2391-2396.

[17] Bai, Zhanbing. "On positive solutions of a nonlocal fractional boundary value problem." Nonlinear Analysis: Theory, Methods \& Applications 72, no.2 (2010): 916-924.

[18] El-Sayed, Ahmed M. A., and Ebtisam O. Bin-Taher. "Positive solutions for a nonlocal multi-point boundary-value problem of fractional and second order." Electronic Journal of Differential Equations 2013, no.64 (2013): 1-8.

[19] Li, Xiuying, and Boying Wu. "Approximate analytical solutions of nonlocal fractional boundary value problems." Applied Mathematical Modelling 39, no.5 (2015): 17171724.

[20] Celiker, Fatih, Bernardo Cockburn, and Ke Shi. "Hybridizable discontinuous Galerkin methods for Timoshenko beams." Journal of Scientific Computing 44, no.1 (2010): 137.

[21] Cockburn, Bernardo, Bo Dong, Johnny Guzman, Marco Restelli, and Riccardo Sacco. "A hybridizable discontinuous Galerkin method for steady-state convection-diffusionreaction problems." SIAM Journal on Scientific Computing 31, no.5 (2009): 38273846 .

[22] Cockburn, Bernardo, and Wujun Zhang. "A posteriori error analysis for hybridizable discontinuous Galerkin methods for second order elliptic problems." SIAM Journal on Scientific Computing 51, no.1 (2013): 676-693.

[23] Nguyen, Ngoc C., and Jaume Peraire. "Hybridizable discontinuous Galerkin methods for partial differential equations in continuum mechanics.” Journal of Computational Physics 231, no.18 (2012): 5955-5988. 
[24] Cockburn, Bernardo, and Kassem Mustapha. "A hybridizable discontinuous Galerkin method for fractional diffusion problems." Numerische Mathematik 130, no.2 (2015): 293-314.

[25] Mustapha Kassem, Maher Nour, and Bernardo Cockburn. "Convergence and superconvergence analyses of HDG methods for time fractional diffusion problems." Advances in Computational Mathematics 42, no.2 (2016): 377-393.

[26] Karaaslan, Mehmet F., Fatih Celiker, and Muhammet Kurulay. “Approximate solution of the Bagley-Torvik equation by hybridizable discontinuous Galerkin methods." Applied Mathematics and Computation 285 (2016): 51-58.

[27] Karaaslan, Mehmet F., Fatih Celiker, and Muhammet Kurulay. "A hybridizable discontinuous Galerkin method for a class of fractional boundary value problems." Journal of Computational and Applied Mathematics 333 (2017): 20-27

[28] Cockburn, Bernardo, Jayadeep Gopalakrishnan, and Raytcho Lazarov. "Unified hybridization of discontinuous Galerkin, mixed, and continuous Galerkin methods for second order elliptic problems." SIAM Journal on Numerical Analysis 47, no.2 (2009): 13191365.

[29] Rehman, Mujeeb, and Rahmat Ali Khan. "A numerical method for solving boundary value problems for fractional differential equations." Applied Mathematical Modelling 36, no.3 (2012): 894-907. 